\title{
Conservative Management of Primary Hyperparathyroidism
}

\author{
P.H. ADAMS, MSc, PhD, FRCP \\ Professor of Medicine, University of Manchester
}

The first description of primary hyperparathyroidism was probably made in 1743[1]. The patient was a woman aged 36 years who complained of pains in the back and limbs and loss of appetite. She was thirsty and passed large amounts of urine. When she died four years later, her bones were found to be soft and full of cysts. This bony condition was almost certainly generalised osteitis fibrosa cystica, a disorder first described by Von Recklinghausen 150 years later. The proof that osteitis fibrosa was causally related to hyperparathyroidism and parathyroid adenoma came in 1926, when Mandel in Vienna removed a parathyroid tumour from a man crippled with this bone disease, and cured his symptoms. Thereafter, other cases of osteitis fibrosa were recognised and treated by parathyroidectomy. Primary hyperparathyroidism was at first thought to be extremely rare, but it soon became apparent that renal stone disease was a much more common mode of presentation. Estimates of the frequency of primary hyperparathyroidism among patients with calcium-containing kidney stones have varied[2], but in patients with calcium oxalate or phosphate stones it is probably 2 to 5 per cent.

In the last 40 years an increasing number of patients with primary hyperparathyroidism have been diagnosed, and most have presented with calcium-containing renal stones. An accompaniment of the increased rate of diagnosis has been the recognition that the disease can present in many ways other than with renal stones and metabolic bone disease (Table 1). A severe and life-threatening

Table 1. The Modes of Presentation of Primary Hyperparathyroidism.

Renal stones and nephrocalcinosis

Osteitis fibrosa cystica, osteoporosis

Pseudo-gout (chondrocalcinosis)

Acute pancreatitis

Peptic ulcer

Depression

Confusional states

Muscle weakness

Hypertension

No symptoms

form of primary hyperparathyroidism (parathyroid crisis) is also known to occur. This condition, which is characterised by severe hypercalcaemia (serum calcium 3.75 to
$5 \mathrm{mmol} /$ litre), is fortunately rare. Although primary hyperparathyroidism is known to present in many different ways, the collected experience of several large clinical series [2-4] indicates that the renal complications are the most common and, perhaps, the most important (Table 2). There is general agreement that some degree of renal damage is common. This is caused by renal calcification (which may be undetectable on radiographs), infection and the complications of renal stones. In some patients this renal damage leads to severe renal failure and hypertension. Although surgical correction of primary hyperparathyroidism cures osteitis fibrosa cystica and appears to reduce the formation of new renal stones[5], it often fails to alter renal function or pre-existing hypertension[3,6]. The ultimate fate of many patients is determined largely by the state of renal function and blood pressure at the time of presentation.

The generally recommended treatment for primary hyperparathyroidism is the surgical removal of all the over-active parathyroid tissue. In the majority of patients the condition arises through adenomatous change in a single gland, and in expert hands surgical treatment is highly effective. Fourman[1] recommended 'that because of the risk of progressive renal damage, operation should not be delayed once the diagnosis has been made'. Many agree with this approach, and there can be little doubt that surgery is the best treatment for many patients. Nonetheless, it should be recognised that this attitude to treatment has evolved from the clinical experience of patients with complications of the disease, and particularly from those presenting with kidney stones. In recent years the mode of presentation of primary hyperparathyroidism has changed, and an increasing number of patients have come to diagnosis without symptoms or obvious complications. This has happened through the development of accurate, and relatively simple, methods for measurement of the serum calcium, and, in particular, through multi-channel biochemical analyses of serum samples. Many of these patients with unsuspected hypercalcaemia have primary hyperparathyroidism. The majority are symptomless, and free of the recognised complications of the disease. This is a general experience, and the proportion of patients with hyperparathyroidism now presenting with renal stones, osteitis fibrosa, and other important complications is substantially less than in the past. This has been the experience at the Manchester Royal Infirmary; in the last 20 years there has been a 
Table 2. Frequency of metabolic bone disease, renal calculus and nephrocalcinosis.

\begin{tabular}{|c|c|c|c|c|c|c|c|}
\hline \multirow[t]{2}{*}{ Author } & \multirow[t]{2}{*}{ No. of patients } & \multicolumn{2}{|c|}{ Bone disease alone } & \multicolumn{2}{|c|}{$\begin{array}{l}\text { Renal stones } \\
\text { and Nephrocalcinosis }\end{array}$} & \multicolumn{2}{|c|}{ Both } \\
\hline & & No. & $\%$ & No. & $\%$ & No. & $\%$ \\
\hline Hellström and Ivemark [3] & 138 & 15 & 10.9 & 76 & 55.1 & 45 & 32.6 \\
\hline Dent [4] & 125 & 27 & 21.6 & 73 & 58.4 & 11 & 8.8 \\
\hline Pyrah et al. [2] & 68 & 17 & 25.0 & 26 & 38.2 & 20 & 29.4 \\
\hline Total & 331 & 59 & 17.8 & 175 & 52.9 & 76 & 23.0 \\
\hline
\end{tabular}

progressive increase in the proportion of patients with unsuspected disease (Fig. 1). Recent studies of primary hyperparathyroidism among a defined urban population

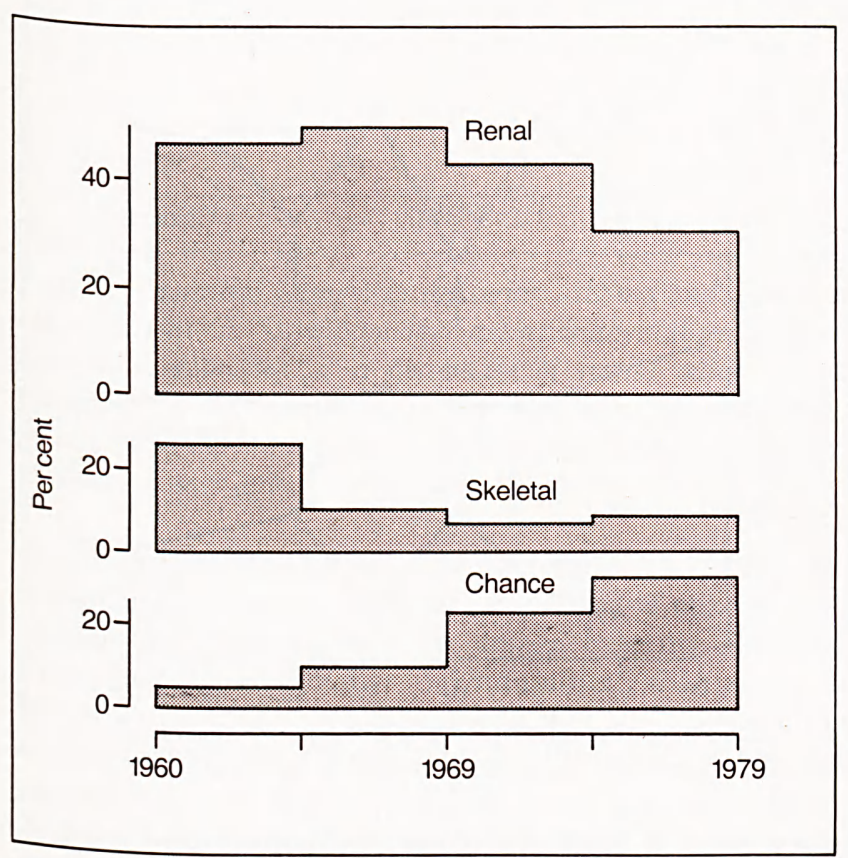

Fig. 1. Proportion of patients with primary hyperparathyroidism presenting with renal stones, bone disease and asymptomatic disease at the Manchester Royal Infirmary, 1960 to 1979. (Data provided by Dr W. P. Stephens.)

indicate that only 4 per cent of patients have kidney stones, 50 per cent are symptomless and free of complications, and primary hyperparathyroidism is more common than formerly believed.

Many patients with unsuspected asymptomatic primary hyperparathyroidism have only a moderate degree of hypercalcaemia[7]; hypercalcaemia of an order that threatens life is rare. The correct treatment for these patients with 'biochemical' as opposed to clinical hyperparathryoidism is uncertain. Some[6,8] consider that, with few exceptions, all patients with hyperparathyroidism should be treated surgically, although others [9] think a conservative approach is justifiable in some patients, provided they are kept under regular review. The rationale for surgical treatment has hitherto included the prevention of important complications, particularly renal damage and associated hypertension. This is a reasonable approach, but it assumes that many, if not all, patients with biochemical hyperparathyroidism are ultimately destined to develop important complications of the disease. The long-term natural history of asymptomatic hyperparathyroidism is not known, and it is well recognised that for many patients with clinical hyperparathyroidism the natural history of the condition can be long and relatively benign. Earlier clinical studies indicate that many patients presenting with kidney stones had a long clinical history before the diagnosis was made[2,3]. Moreover, the frequency of renal stone formation varies considerably among individual patients. Few studies have been made of the natural history of asymptomatic (biochemical) hyperparathyroidism. The extensive studies made at the Mayo Clinic[10,11,13], and a brief report from Leeds[12] seem to show that the condition is relatively benign.

In the last twenty years, over 200 patients with primary hyperparathyroidism have been seen at the Manchester Royal Infirmary. Most have presented with kidney stones (see Fig. 1) and many have been treated by surgery. A small number of patients, with mild or asymptomatic disease, have been treated conservatively. Thirty-one of these conservatively managed patients have been observed for periods of 1 to 12 years (mean 4 years); 9 have been followed for at least 5 years. A formally planned prospective trial has not been done, but the observations provide further information on the natural history of asymptomatic hyperparathyroidism and mild clinical forms of the disease.

Two groups of patients have been studied, one with clinical hyperparathyroidism, in which the patients presented with typical features of the disease (mainly renal stones); and the other with asymptomatic (biochemical) hyperparathyroidism in which the diagnosis had been made by chance. Two patients (one in each group) had previously had unsuccessful neck operations. The diagnosis of primary hyperparathyroidism was made on clinical, biochemical and radiological grounds.

The decision to follow a conservative approach was based on clinical considerations (age, general medical status, the presence or absence of complications), and on two arbitrary criteria. These criteria were that the serum calcium had to be less than $3.0 \mathrm{mmol} /$ litre and the 24 hour urinary calcium less than $10 \mathrm{mmol}$. Most patients were assessed on one or more occasions in a metabolic unit and were followed regularly in the out-patient clinic. Serial measurements were made of the serum calcium, inorganic phosphate, creatinine, urea and alkaline phosphatase; and particular attention was given to the development of hypertension, renal stones and bone disease. 
Table 3. Clinical and biochemical features of patients treated conservatively. Numbers in parentheses are numbers in each group; values expressed as mean \pm SE. Serum calcium is first summer value.

\begin{tabular}{|c|c|c|c|c|c|}
\hline CLINICAL & $\begin{array}{c}\text { Male } \\
(7)\end{array}$ & $\begin{array}{c}\text { Female } \\
(5)\end{array}$ & $\begin{array}{c}\text { Serum } \\
\text { Calcium* } \\
\text { (mmol/litre) }\end{array}$ & $\begin{array}{c}\text { Serum } \\
\text { Creatinine } \\
(\mu \mathrm{mol} / \mathrm{litre})\end{array}$ & $\begin{array}{c}\text { Serum } \\
\text { Parathyroid hormone } \\
(\mu \mathrm{g} / \text { litre })\end{array}$ \\
\hline Age (yr) & $48 \pm 3.84$ & $62.8 \pm 4.49$ & & & \\
\hline Range & $32-61$ & $46-71$ & $\begin{array}{c}(12) \\
2.77 \pm 0.031\end{array}$ & $\begin{array}{c}(12) \\
87.3 \pm 4.11\end{array}$ & $\begin{array}{c}(12) \\
0.99 \pm 0.21\end{array}$ \\
\hline ASYMPTOMATIC & (3) & (16) & (18) & (19) & (15) \\
\hline Age $(y r)$ & $52 \pm 13.58$ & $59.1 \pm 2.23$ & $2.84 \pm 0.025$ & $82.83 \pm 4.75$ & $1.21 \pm 0.109$ \\
\hline Range & $36-79$ & $45-71$ & & & \\
\hline
\end{tabular}

Table 3 shows the clinical details of the two groups when they first presented with hypercalcaemia. The patients with asymptomatic hyperparathyroidism were mainly middle-aged and elderly women, the characteristic age and sex distribution of biochemical hyperparathyroidism and hyperparathyroidism in general[2,7]. The majority of the clinical group presented with renal stones. The most common primary diagnosis in the biochemical group was hypertension. Eight of this group (six with hypertension) were receiving thiazide diuretics when hypercalcaemia was first discovered.

\section{Sequential Changes}

Serum Calcium. Minor variations in the serum calcium concentration were observed in many patients and these were probably methodological in origin. In some patients, more distinct fluctuations in the serum calcium values were observed and these were clearly related to the season of the year. In these patients the serum calcium was higher in the summer than in the winter. An example of this seasonal phenomenon is shown in Fig. 2, which also shows that there was a reverse pattern of change in the serum parathyroid hormone concentration. Although no direct assessments of vitamin D status were made, these seasonal variations almost certainly arise through seasonal variations in the biosynthesis of vitamin $D$. In normal persons, the serum concentration of 25-hydroxyvitamin D (a measure of vitamin D status) is highest during the summer, and falls progressively during the winter. Patients with primary hyperparathyroidism who habitually avoid sunshine can develop vitamin D deficiency and osteomalacia; in these circumstances the serum calcium concentration falls, and it increases once the deficiency is relieved[14]. It is important to recognise this seasonal variation in the serum calcium, particularly when the decision to adopt a conservative approach is based on somewhat arbitrary criteria. Otherwise, the normal summer increase might be mistakenly attributed to the start of an inexorable rise in the serum calcium concentration. A real increase in the serum calcium during the winter is likely to be of much greater significance. Because of these effects the sequential changes in the serum calcium (Fig. 3) refer only to the summer values. The findings in both groups of patients were the same, and the combined results are shown; on average,

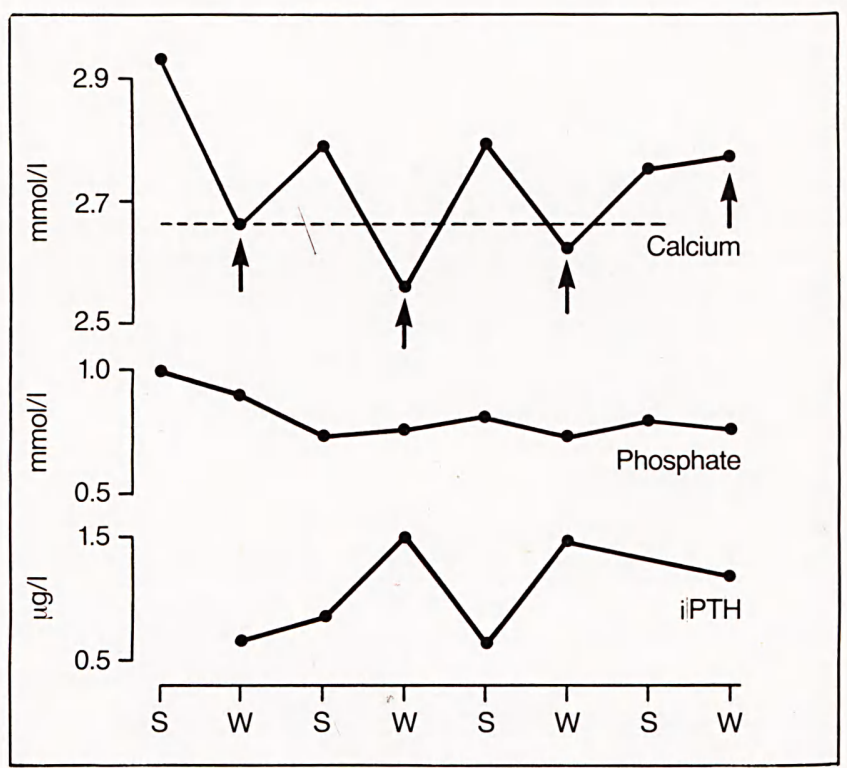

Fig. 2. Seasonal variations in the serum concentrations of calcium and parathyroid hormone (iPTH) in a patient with asymptomatic hyperparathyroidism. $S=$ Summer; $W=$ Winter.

there was no significant change in the serum calcium concentration with time. Three patients did not conform to this general pattern and developed significant increases in the serum calcium; the reasons for these changes were different in each case. Their case histories are briefly described.

Patient 1. This patient was referred to the metabolic unit in 1973. She was then 69 years old and had a previous history of backache (lumbar spondylosis), peptic ulcer and recurrent urinary infections. She became hypertensive, and was later found to have hypercalcaemia on routine biochemical screening. She had no kidney stones and no radiological evidence of nephrocalcinosis or osteitis fibrosa; the urinary calcium excretion was not excessive $(4 \mathrm{mmol} /$ day, $160 \mathrm{mg} /$ day $)$ but the creatinine clearance was reduced $(43 \mathrm{ml} / \mathrm{min})$. The initial serum calcium was $2.76 \mathrm{mmol} /$ litre. A conservative approach was adopted. Figure 4 shows the sequential changes in the serum calcium concentration. She first attended in Janu- 

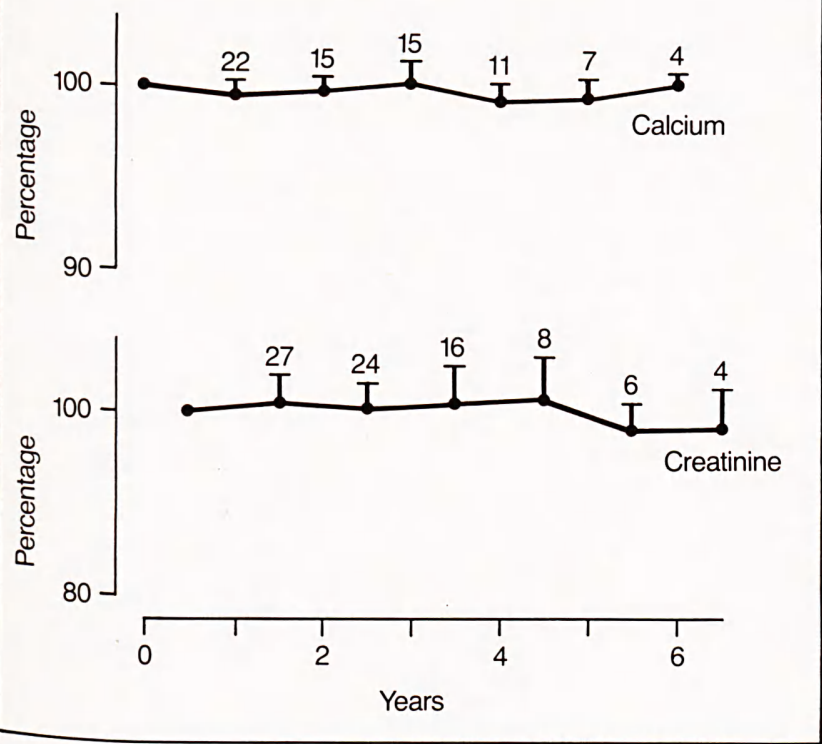

Fig. 3. Sequential changes in the serum calcium and creatinine concentrations in asymptomatic hyperparathyroidism. Serum calcium is expressed as a percentage of initial value, serum creatinine is the average value for each year expressed as a percentage of the mean value of the first year. Numbers represent numbers in each group. Mean and SE shown.

ary 1973, and the serum calcium increased during the following summer and decreased during the next winter. During the next summer the serum calcium increased again, but was now higher $(3.1 \mathrm{mmol} / \mathrm{litre})$ than at any of her previous visits. We discovered that she had recently started a thiazide diuretic for hypertension. This was

Fig. 4. Patient 1. Sequential changes in serum calcium and creatinine concentrations.

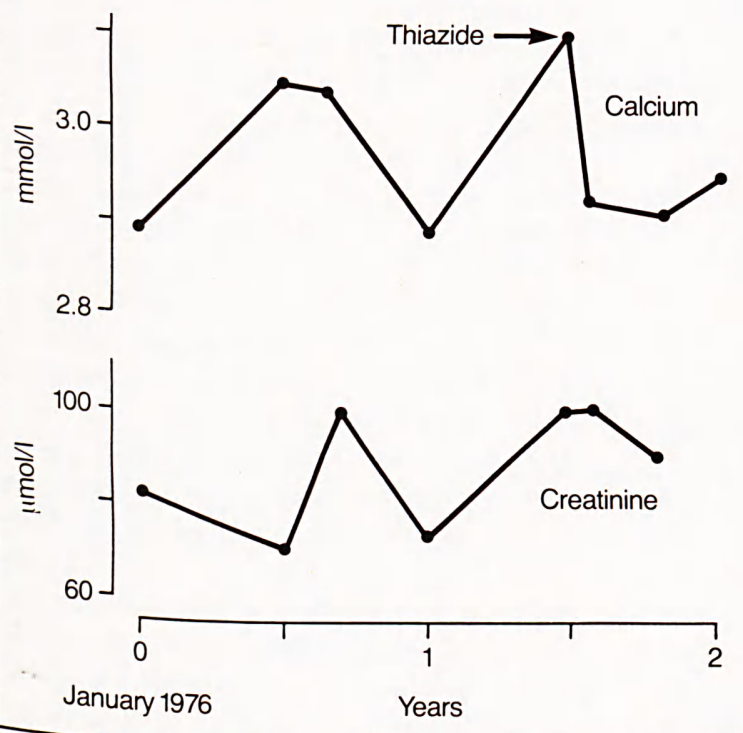

stopped and another hypotensive drug was substituted; the serum calcium decreased to the more usual level. This apparently abnormal increase in the serum calcium was attributed to the effects of the diuretic, but with hindsight (since it was not recognised at the time) some of the variation was probably related to seasonal influences.

The effects of thiazide diuretics on calcium metabolism are well documented, but they seem not to be widely appreciated. Thiazide diuretics increase the renal tubular reabsorption of calcium and promote the entry of calcium into the extracellular space[15]. This effect seldom causes hypercalcaemia in normal persons, because serum calcium homeostasis is maintained through the action of normally functioning parathyroid glands. Patients with primary hyperparathyroidism, and hence with largely autonomous parathyroid glands, behave differently. Parathyroid hormone secretion is not reduced in response to increases in the serum calcium concentration[15]; and, as a result, the serum calcium increases significantly. Withdrawal of a thiazide diuretic in primary hyperparathyroidism is accompanied by a decrease in the serum calcium and an increase in the urinary calcium but the serum concentration of parathyroid hormone is unchanged[15]. Thiazide diuretics should be withdrawn if the clinical situation allows, because this manoeuvre might reduce the serum calcium concentration to a level that is appropriate for conservative management[15]. Moreover, because thiazides tend to exacerbate hypercalcaemia in primary hyperparathyroidism they should be avoided or used cautiously in patients known to have the disease.

Patient 2. This patient was referred to the metabolic unit in 1971. She was then aged 69 years. She had presented in 1968, at another hospital, complaining of backache; she was found to have hypercalcaemia and hypophosphataemia but no action was taken. Two years later she developed hyperthyroidism and was found to have hypertension; she was treated with carbimazole and a thiazide diuretic. Hypercalcaemia was again noted. When she was referred to the metabolic unit in 1973, the serum calcium was $2.81 \mathrm{mmol} /$ litre. She was euthyroid and had no kidney stones or osteitis fibrosa; a diagnosis of asymptomatic hyperparathyroidism was made. The carbimazole was continued but the thiazide diuretic was stopped. A conservative approach was adopted. Her subsequent progress was satisfactory and she remained euthyroid. The serum calcium remained relatively stable (Fig. 5) until the carbimazole was stopped in October 1976. Two months later the serum calcium concentration was higher than it had been previously $(3.12 \mathrm{mmol} / \mathrm{litre})$, and it was at this level a few weeks later. She now had all the features of hyperthyroidism (serum thyroxine $189 \mathrm{nmol} / \mathrm{litre}$ ). In this patient the observed increase in the serum calcium occurred in the winter, and was related to the hyperthyroidism, a condition known to cause hypercalcaemia[16] and to co-exist with primary hyperparathyroidism.

Patient 3. This patient was referred in 1971. She was then aged 64 years. She had passed renal stones during pregnancies when she was 32 and 35 years old. She had 


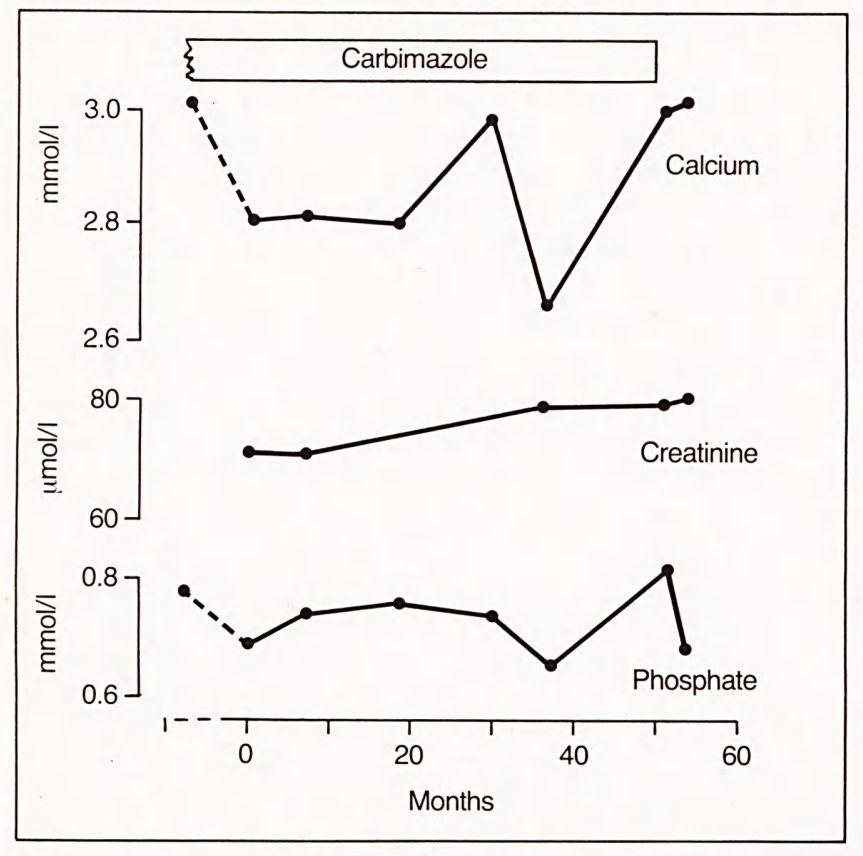

Fig. 5. Patient 2. Sequential changes in serum calcium, creatinine and phosphate concentrations.

no more attacks and remained well for the next 30 years. When she was 64 years of age she had a mild cerebral thrombosis, and she was found to have moderately severe hypertension and hypercalcaemia. She was shown to have a stone in the left kidney and infected urine; there was no osteitis fibrosa. The serum calcium was $2.85 \mathrm{mmol} / \mathrm{litre}$. The urinary calcium excretion was excessive $(15.7 \mathrm{mmol} /$ $24 \mathrm{hr} ; 628 \mathrm{mg}$ ), but renal function was reasonably well preserved (creatinine clearance $75 \mathrm{ml} / \mathrm{min}$ ). She was reluctant to undergo parathyroid surgery, and in view of the hypertension and recent stroke a conservative approach was adopted. Figure 6 shows her subsequent progress. The hypertension was somewhat difficult to control, but the serum concentrations of calcium and creatinine remained relatively stable for three years. In October 1975, four years after her first attendance, the hypertension was poorly controlled (BP 190/120) and the serum calcium had increased substantially $(3.26 \mathrm{mmol} /$ litre). She was re-admitted. Renal function had deteriorated (creatinine clearance $53.8 \mathrm{ml} / \mathrm{min}$ ) but the renal stone had not changed in size. She was treated with propranolol and underwent surgery. A large single parathyroid adenoma was removed. The serum concentrations of calcium and parathyroid decreased to normal, but there was no sustained improvement in the hypertension or renal function.

This is the only patient who has required parathyroid surgery, and in whom worsening hypercalcaemia and renal function could be confidently attributed to the parathyroid disease. This patient had almost certainly had primary hyperparathyroidism for nearly 40 years, but the reason she ultimately deteriorated and developed more severe hypercalcaemia is uncertain. One explanation could be that her ability to excrete calcium through the kidneys was compromised by progressive renal impairment induced by sustained hypertension and chronic renal infection. Another is that the parathyroid tumour increased in size and activity. The serum concentration of parathyroid hormone was always raised, and it increased further up to the time of operation (Fig. 6). This finding

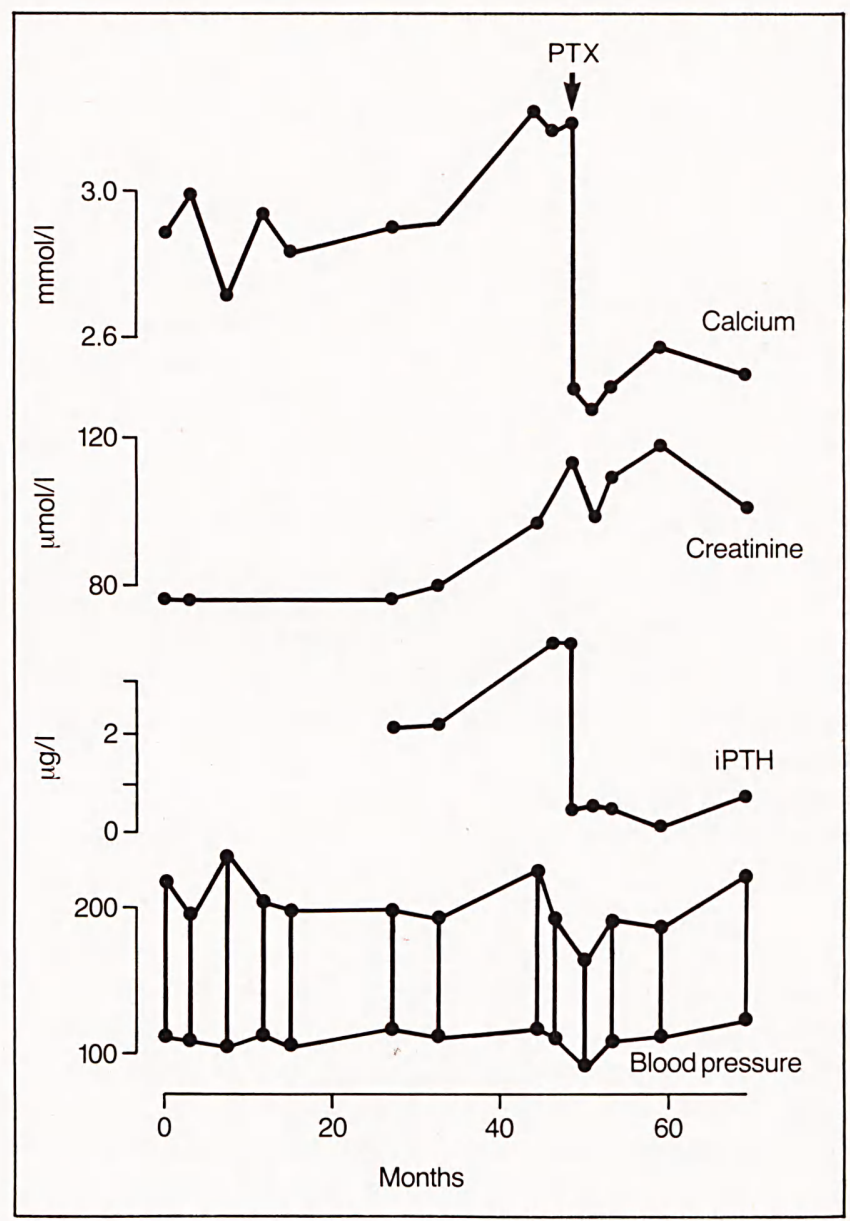

Fig. 6. Patient 3. Sequential changes in serum calcium, creatinine, and parathyroid hormone concentrations. $P T X=$ parathyroidectomy.

suggests that the parathyroid adenoma had, in fact, become more active, although a similar upward trend in the serum parathyroid hormone concentration has not been a consistent feature of the other patients studied.

Serum Creatinine. There was some individual variation in the sequential changes in the serum creatinine but, on average, the mean values were relatively stable and showed no upward trend with time (see Fig. 3). In the 9 patients followed for at least 5 years there was no significant difference between the mean serum creatinine value of the first and the last year of observation.

One patient appeared to show a progressive increase in the serum creatinine with time (Fig. 7). This patient, an elderly woman who has been followed for 10 years, had had a nephrectomy for kidney stones when she was 58 years old. The serum calcium has remained relatively stable over the years and she has developed no further kidney stones. Analysis of the data shows that there has been a significant upward trend in the serum creatinine 


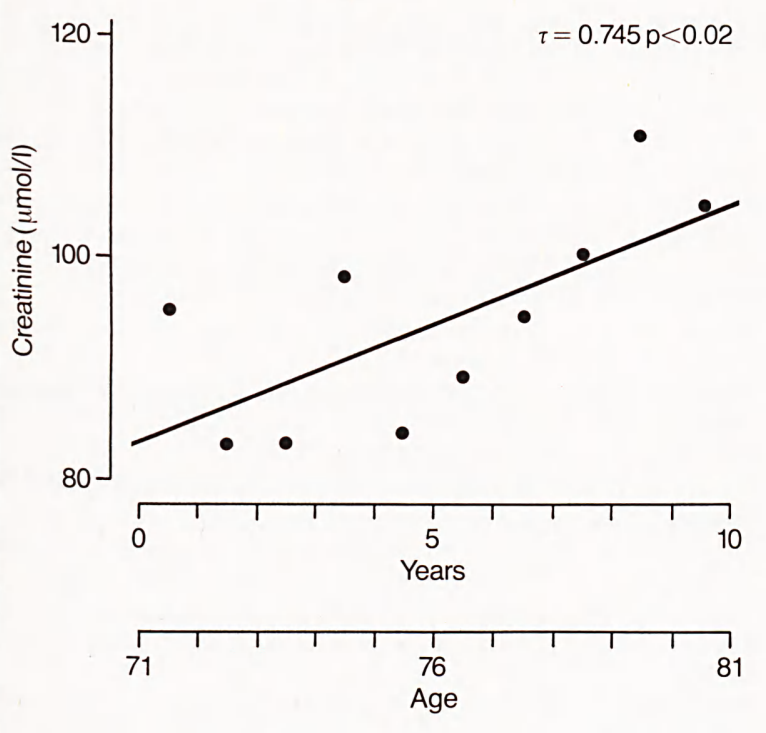

Fig. 7. Sequential changes in the serum creatinine concentration in an elderly woman with primary hyperparathyroidism. Values are the means for each year of observation.

with time, but the latest serum creatinine is not abnormal (88 $\mu \mathrm{mol} / \mathrm{litre})$. This pattern of change might not be entirely related to her primary hyperparathyroidism. This patient is now 81 years old. In recent years she has developed heart failure and been treated with frusemide. Heart failure, the diuretic therapy and the effects of ageing might have contributed to the observed deterioration in renal function.

None of the patients studied has developed life-threatening acute hypercalcaemia (parathyroid crisis) or acute pancreatitis and, apart from the four patients already described in detail, none has shown any substantial change in the serum calcium or creatinine concentrations with time. None of the patients with asymptomatic (biochemical) hyperparathyroidism has developed renal stones, nephrocalcinosis or osteitis fibrosa cystica. Two patients, both elderly women, have vertebral fractures; but both have extensive osteitis deformans of the spine.

\section{Conservative Management or Not?}

The findings in the present study are in broad agreement with those made at the Mayo Clinic[11], namely, that many patients with mild and asymptomatic primary hyperparathyroidism show little alteration in the serum calcium concentration and in renal function with time. Likewise, no criterion has been identified at first presentation which distinguishes between those patients who are destined to require surgery and those who are not. The Mayo Group found that the careful surveillance of their patients was time-consuming, expensive and taxing. They also found that with time some of their patients failed to attend for review; they are now inclined to recommend surgery for asymptomatic hyperparathyroidism, unless the patient is prepared to attend regularly for follow-up. In a later publication from the Mayo Clinic, the financial cost to the patient of five years of follow-up was found to be greater than if they had undergone surgery in the first instance. Some of these considerations are applicable to clinical practice in the UK. Coe and Favus[17] suggested that the management of asymptomatic hyperparathyroidism should be subjected to a prospective therapeutic trial testing the benefits of surgery and determining whether untreated patients are at greater risk of progressive renal impairment, hypertension and other problems. Until such a trial is done, they recommended parathyroidectomy for all patients. There is much to be said for this argument, but a conservative approach would seem reasonable in some patients, particularly the elderly. Younger patients present more difficulty, because a conservative approach is likely to entail decades of regular clinical surveillance that may well induce needless anxiety. Moreover, the available evidence in favour of a conservative approach is based on a relatively short experience (five years), or, if longer, on relatively few patients. Younger patients might be vulnerable to hitherto unrecognised complications in the long term.

Although many patients with asymptomatic hyperparathyroidism seem to be at little risk of severe hypercalcaemia and progressive renal impairment, the long-term effect of sustained hyperparathyroidism on the skeleton and the development of clinical osteoporosis is uncertain. Parathyroid hormone is a potent stimulator of bone resorption, and many patients without clinical evidence of bone disease have microscopic evidence of increased bone resorption[18]. The effect of parathyroid hormone seems to be exaggerated by oestrogen deficiency, and this is thought to explain the accelerated bone loss that normally accompanies the menopause[19], and predisposes to osteoporosis. Patients with primary hyperparathyroidism might be at risk of excessive losses of bone at all ages, and, in women, particularly after the menopause. Primary hyperparathyroidism is a disease mainly of middle-aged women[2,3]. There is clear evidence that many patients have less bone than normal for their age[20-22], and by implication lose bone at an accelerated rate. Moreover, sequential studies indicate that the deficit in bone mass is only partially regained after surgical correction of the disease[22,23]. This deficit, which is largely permanent, might predispose to clinical osteoporosis at a later age. There is some evidence that spinal osteoporosis with crush vertebral fractures is more common among affected patients than in normal persons of the same age[24]. This has not been a particular feature of our patients, but this aspect certainly warrants further study.

Apart from the possible deleterious effects on the skeleton, sustained hyperparathyroidism might have adverse effects on other organs and systems. In an elderly group of patients, some of whom had mild hyperparathyroidism and none of the well-recognised complications of the disease, the major complaints were general malaise, immobility, muscle weakness and cerebral impairment[25]. In these patients parathyroidectomy restored mobility and muscle strength, improved cerebral function and conferred a general feeling of well-being. Some of 
these symptoms are non-specific and not generally attributed to hyperparathyroidism; they are difficult to assess objectively, but the observed improvements could represent specific responses to treatment. Primary hyperparathyroidism is also associated with disturbances of the cardiovascular system. The association between primary hyperparathyroidism and hypertension is well recognised; in some patients this is clearly related to renal damage[3]. Hypertension seems to occur more commonly among patients with asymptomatic hyperparathyroidism than in normal persons of the same age[7]. Some of the patients reported here presented with hypertension. Whether hypertension should be regarded as a complication of hyperparathyroidism in these asymptomatic patients is uncertain. Primary hyperparathyroidism occurs commonly at an age when hypertension is also common; the association might be coincidental. Nonetheless, hyperparathyroidism could have a bearing on the severity and age of onset of hypertension. The response of hypertension to parathyroidectomy is generally unfavourable [3,6], and hypertension alone is not an indication for surgery.

When all these considerations are taken into acount, it could well be argued that, despite the apparently good prognosis, surgery is the treatment of choice for the younger patient. Parathyroid surgery is relatively safe, and in expert hands at least 95 per cent of patients require only one operation for cure[26]. If this policy were to be generally adopted for the treatment of mild asymptomatic hyperparathyroidism, two essential conditions would have to be satisfied. The first is that the diagnosis must be established beyond reasonable doubt. Patients with asymptomatic hyperparathyroidism can be difficult to differentiate from patients with familial hypocalciuric hypercalcaemia, for whom parathyroid surgery is seldom indicated[27]. The second condition is that the surgery should be done by those well experienced in this work. Even when both conditions have been satisfied, occasional unforeseen misfortunes have occurred and have proved fatal[28]. Such happenings are rare, but serve to remind us that the correct choice of treatment for asymptomatic primary hyperparathyroidism requires careful thought.

\section{Acknowledgement}

This work was supported in part by a grant from the Medical Research Council and DHSS to Professor S. W. Stanbury.
This article is based on a paper read at the College Regional Conference in Manchester in September 1981.

\section{References}

1. Fourman, P. and Royer, P. (1968) Calcium Metabolism and the Bone. Oxford and Edinburgh: Blackwell Scientific Publications.

2. Pyrah, L. N., Hodgkinson, A. and Anderson, C. K. (1966) British Journal of Surgery, 53, 245.

3. Hellström, J. and Ivemark, B. I. (1962) Acta Chirurgica Scandinavica, Suppl. 294.

4. Dent, C. E. (1962) British Medical Journal, 2, 1419, 1495.

5. McGeown, M. G. (1961) Lancet, 1, 586.

6. Britton, D. C., Thompson, M. H., Johnson, I. D. A. and Fleming, L. B. (1971) Lancet, 2, 74.

7. Heath, H., Hodgson, S. and Kennedy, M. A. (1980) New England Journal of Medicine, 302, 189.

8. Wilkinson, R. (1981) Hospital Update, 7, 801.

9. Martin, T. J. and Larkins, R. G. (1981) Medicine International (UK edition), 1, 267.

10. Purnell, D. C., Smith, L. H., Scholz, D. A., Elveback, L. A. and Arnaud, C. D. (1971) American Journal of Medicine, 50, 670.

11. Purnell, D. C., Scholz, D. A., Smith, L. H., Sizemore, G. W., Black, B. M., Goldsmith, R. S. and Arnaud, C. D. (1974) ibid., 56,800 .

12. Hodgkinson, A., Peacock, M. and Nordin, B. E. C. (1971) Lancet, 2,49 .

13. Keating, F. R. (1970) Medical Clinics of North America, 54, 511.

14. Stanbury, S. W. (1981) Journal of the Royal College of Physicians of London, 15, 205.

15. Klimiuk, P. S., Davies, M. and Adams, P. H. (1981) Postgraduate Medical Journal, 57, 80.

16. Adams, P. H., Jowsey, J., Kelly, P. J., Riggs, B. L., Kinney, V. R. and Jones, J. D. (1967) Quarterly Journal of Medicine, 36, 1.

17. Coe, F. L. and Favus, M. J. (1980) New England Journal of Medicine, $302,224$.

18. Riggs, B. L., Kelly, P. J., Jowsey, J. and Keating, F. R. (1965) Journal of Clinical Endocrinology and Metabolism, 25, 77.

19. Heaney, R. P. (1965) American Journal of Medicine, 39, 877.

20. Forland, M., Strandjord, N. M., Paloyan, E. and Cox, A. (1968) Archives of Internal Medicine, 122, 236.

21. Dalén, N. and Hjern, B. (1974) Acta Endocrinologica, 75, 297.

22. Ringe, J. D., Kruse, H. P. and Kubhlencordt, F. (1980) Proceedings of Fourth International Conference on Bone Measurement. NIH Publication 80-1938.

23. Parfitt, A. M., Rao, D. S., Kleerekoper, M., Walczak, N., Levin, N., Oliver, I. and Frame. B. (1980) Proceedings of Fourth International Conference on Bone Measurement. NIH Publication 80-1938.

24. Dauphine, R. T., Riggs, B. L. and Scholz, D. A. (1975) Annals of Internal Medicine, 83, 365.

25. Heath, D. A., Wright, A. D., Barnes, A. D., Oates, G. D. and Durricott, N. J. (1980) British Medical Journal, 280, 1406.

26. Satava, R. M., Beahrs, O. H. and Scholz, D. A. (1975) Archives of Surgery, 110, 625.

27. Davies, M., Klimiuk, P. S., Adams, P. H., Lumb, G. A., Large, D. M. and Anderson, D. C. (1981) British Medical Journal, 282, 1023.

28. Gough, M. H., Smith, F. and Bishop, M. C. (1971) Lancet, 1, 1178. 